\title{
Investigation on the intermolecular interactions in aliphatic isocyanurate liquids: revealing the importance of dispersion
}

\author{
Veniero Lenzi $^{\mathrm{a}, *}$, Piet J. Driest ${ }^{\mathrm{b}, \mathrm{c}}$, Dirk J. Dijkstra ${ }^{\mathrm{b}}$, Marta M.D. Ramos ${ }^{\mathrm{a}}$, Luís S.A. Marques ${ }^{\mathrm{a}}$ \\ a Department/Centre of Physics, University of Minho, Campus de Gualtar, Braga 4710-057, Portugal \\ ${ }^{\mathrm{b}}$ Covestro Deutschland AG, CAS-Global REDD, Leverkusen 51365, Germany \\ ${ }^{\mathrm{C}}$ MIRA Institute for Biomedical Technology and Technical Medicine, Department of Biomaterials Science and Technology, University of Twente, P.O. Box 217, Enschede 7500 AE, \\ the Netherlands
}

\section{A R T I C L E I N F O}

\section{Article history:}

Received 20 November 2018

Received in revised form 29 January 2019

Accepted 31 January 2019

Available online 7 February 2019

\section{Keywords:}

Isocyanates

Isocyanurates

Viscosity

Dispersion

Ab-initio methods

Molecular dynamics

\begin{abstract}
A B S T R A C T
Aliphatic isocyanurates are nowadays used routinely in the development of advanced materials like polyurethane nanocomposites and 3D-printed components, due to their versatile reactivity and the good mechanical and optical properties they confer to the final material. In these applications, a control of the properties at the micro- and nanoscale is desired, therefore a deep understanding of intermolecular interactions is required. Using ab-initio calculations and molecular dynamics simulations, the intermolecular interactions of aliphatic isocyanurates are investigated in detail. The presence of an isocyanate-isocyanurate interaction is demonstrated, and the strong dispersion character of isocyanurate-based interactions is revealed. Calculations of the free energy of binding of the different interactions in gas and liquid phase are provided. The microscopic structure of aliphatic functional and non-functional isocyanurates is analysed and related to the different interaction types, and finally their possible relationship with macroscopic dynamic variables is discussed.
\end{abstract}

(c) 2019 Elsevier B.V. All rights reserved.

\section{Introduction}

Isocyanates are one of the most important families of molecules in modern chemistry. Long-time known for their role as polyurethane building blocks [1], their peculiar chemistry and reactivity makes them very appealing for other applications, such as surfaces and nanocarriers functionalization [2,3,4,5], stable matrix for non-linear optically (NLO) active devices [6], design of complex surfaces [7], synthesis of artificial proteins [8], and their use in this sense is expected to grow as new synthesis routes involving isocyanates $[9,10]$ and novel processing techniques, such as molecular layer deposition [11], are developed.

Remarkably, isocyanates play a prominent role in graphene based nanocomposites, as they are used to functionalize graphene oxide [12], making it possible to join the very interesting properties of graphene with the versatility of isocyanate chemistry. In this sense, isocyanate-functionalized graphene oxide was used to craft flexible energy storage devices [13] and nanocatalysts [14]. These molecules

\footnotetext{
* Corresponding author.

E-mail address: veniero.lenzi@fisica.uminho.pt (V. Lenzi).
}

have received also interest in the field of reactive inkjet 3D printing [15], with potential application for biomaterials [16]. Moreover, if nanocomposite-based reactive inks could be used, this would lead to the development of printable devices with tunable properties and functionality.

For such purposes, a good candidate amidst others is represented by aliphatic trifunctional isocyanurates, obtained by trimerization reaction of linear diisocyanates (Fig. 1). They combine the possibility to control the viscosity of precursors during processing with a high crosslinking capability, in addition to good optical and mechanical properties [17,18,19,20,21,22], which were exploited to build shape memory materials for bioapplications [23].

In a previous work [24], the viscosity of various aliphatic isocyanurate liquids, both functional and non-functional(i.e. without any free NCO groups) was measured. It was shown that the viscosity of functional isocyanurates is at least three orders of magnitude larger than that of their diisocyanate precursors (e.g. $614 \mathrm{mPa} \cdot \mathrm{s}$ for HDI trimer [24] compared to $2.3 \mathrm{mPa} \cdot \mathrm{s}$ for HDI [25]) and increasing as the alkylene chain-length between the isocyanate and isocyanurate decreases. Conversely, a weak dependence on alkylene chain length was found in the case of non-functional molecules, and smaller viscosities $(200 \mathrm{mPa} \cdot \mathrm{s})$ were found. Even though this behaviour was 
a<smiles>CPN=C=O</smiles>

b<smiles>O=C=NPN=C=O</smiles>

Fig. 1. Representation of an: isocyanate (a), diisocyanate(b) and a trifunctional isocyanurate (c), also referred to as (isocyanate) trimer. Non-functional isocyanurates are equivalent to (c), except that the terminal $\mathrm{NCO}$ groups are substituted by $-\mathrm{CH}_{3}$ terminations. In all schemes $\mathrm{R}=-\left(\mathrm{CH}_{2}\right)_{n}-$.

explained by hypothesizing the presence of a previously unnoticed isocyanate-isocyanurate ring interaction, the details of its cause and nature remain unclear.

This study aims to investigate the intermolecular interactions of aliphatic isocyanurates from a fundamental level, using ab-initio methods such as Density Functional Theory (DFT) and Symmetry Adapted Perturbation Theory (SAPT), in order to properly characterize them and define their origin. In addition, the local structure properties of isocyanurate liquids are studied by means of Molecular Dynamics (MD) simulations, so that their microscopic features can be revealed.

\section{Methods}

DFT, MP2 and $\operatorname{CCSD}(\mathrm{T})$ calculations were performed using the Gaussian 09 code [26]. Concerning DFT, the B3LYP [27] and the APFD [28] hybrid functionals were used, chosen because widely applied for organic molecules and capable to treat dispersion forces. In the first case, the Grimme's D3 empirical dispersion correction, with BeckeJohnson damping [29,30], was included. In APFD, the empirical dispersion is already embodied in the functional. Post Hartree-Fock calculations were performed using the Dunning's augmented correlation consistent basis sets [31]. A Gaussian triple-zeta basis set with polarizable and diffuse orbitals $[32,33,34] 6-311++G(d, p)$ was preferred to the aug-cc-pVTZ set in all DFT calculations. All geometrical optimizations were conducted using tight convergence criteria, corresponding to a force threshold of $0.012 \mathrm{kcal} /(\mathrm{mol} \cdot \AA)$ per atom. The dimer interaction energy is defined as $E_{\text {int }}=E_{A B}-E_{A}-E_{B}$, where $E_{A B}$ is the complex energy in the optimized geometry and the monomer energies $E_{A}$ and $E_{B}$ are calculated without any further optimization. All $E_{\text {int }}$ calculations are counterpoise-corrected [35]. SAPT [36] calculations were performed using the Psi4 code [37] on B3LYP-D3BJ/6-311++G(d,p) optimized structures, using Dunning's double zeta basis sets. The non-covalent interaction $\mathrm{NCI}$ analysis [38] was performed with the MultiWFN software package [39], used to analyse and elaborate the electron densities obtained from B3LYP calculations. The NCI method is based upon the analysis of the reduced density gradient $\mathrm{s}$, defined as:

$s=\frac{1}{2\left(3 \pi^{2}\right)^{1 / 3}} \frac{|\nabla \rho|}{\rho^{4 / 3}}$, $\rho$ being the electronic density, and identifies the non-covalent interaction by the presence in $s(\rho)$ of regions in which both the density $\rho$ and its second eigenvalue $\lambda_{2}$ are small. $s(\rho)$ isosurfaces can be plotted and coloured according to $\operatorname{sgn}\left(\lambda_{2}\right) \rho$, allowing the visualization of non-covalent interaction sites.

Molecular dynamics simulations were performed using LAMMPS [40]. Isocyanates and related molecules were modelled using a specifically developed GAFF-based force field [41]. For free energy calculations, the potential of mean force (PMF) was estimated through the adaptive biasing force $(\mathrm{ABF})$ method using the collective variable COLVARS [42] module, distributed within LAMMPS. To simulate the gas phase, molecules were put in a very large box (200 length), without periodic boundary conditions, using a cutoff for all pair forces of $80 \AA$, a Langevin thermostat with a damping constant of $5 \mathrm{ps}^{-1}$ and a timestep of $1 \mathrm{fs}$. The gas-phase free energies were calculated from $1 \mu$ s simulation runs. Liquid simulations were run under NPT conditions. Periodic boxes containing 343 molecules were equilibrated for $100 \mathrm{ps}$ at $298.15 \mathrm{~K}$ and $1 \mathrm{~atm}$, with a $1 \mathrm{fs}$ timestep, using a Nosé-Hoover thermostat [43] and barostat [44] with damping constants of 0.1 and $1 \mathrm{ps}^{-1}$, respectively. All pair forces were calculated directly using a cutoff radius of $13 \AA$ A. Long-range forces were treated using the Particle-Particle-Particle Mesh (P3M) method, applied for both electrostatic [45] and Van der Waals [46] interactions. Concerning $\mathrm{ABF}$ liquid calculations, the collective variable was split in two non-overlapping windows of $7.5 \AA$ each, and 50 ns of simulation per window were run, meaning a total of $100 \mathrm{~ns}$ of simulation time per $\mathrm{ABF}$ calculation. To perform the local structure analysis, data were sampled each 20 ps from a 5 ns production run under NVE conditions, starting from NPT-equilibrated systems at 298.15 K and $1 \mathrm{~atm}$. All MD results were obtained as averages over time and three independent production runs.

\section{Results and discussion}

\subsection{Ab-initio study of isocyanate and isocyanurate intermolecular interactions}

\subsubsection{Assessment of the accuracy of ab-initio methods for isocyanates and isocyanurates}

Throughout this work, DFT and SAPT methods were used to calculate the interaction energies $\left(E_{\text {int }}\right)$ of isocyanate and isocyanurate dimers. It is important to have an idea on the accuracy of these methods for such molecules. For this purpose, a test set of 16 small molecule dimers, built starting from methane, isocyanic acid (IC), methyl isocyanate (MIC) and formaldehyde, was introduced. An isocyanuric acid-methane dimer was also included to consider dispersion ring-based interactions. Optimized structures at the mp2/augcc-pVTZ level of theory, which can be found in Supplementary Material 2, were used to calculate the reference interaction energies, obtained with the $\operatorname{CCSD}(\mathrm{T})$-complete basis set extrapolation scheme as described in Ref. [47]. An exception was represented by the isocyanuric acid-methane dimer, for which the $\operatorname{CSD}(\mathrm{T})-\mathrm{cbs}(\mathrm{aDZ})$ [47] method was preferred due to limits in available computational power. All results are shown in Fig. 2.

Concerning DFT methods, the B3LYP-D3BJ and the APFD hybrid functionals were compared using the $6-311++G(d, p)$ basis set, and showed similar performance. APFD, with a mean absolute error (MAE) of $0.16 \mathrm{kcal} / \mathrm{mol}$, behaved slightly better than B3LYP-D3BJ, that scored a MAE of $0.20 \mathrm{kcal} / \mathrm{mol}$. However, it showed a slight tendency to overestimate the reference $E_{\text {int }}$ throughout all the set, particularly evident for the ICU-CH4 dimer. To further compare the methods, the S22 $[47,48]$ benchmark was performed, revealing that B3LYP-D3BJ $/ 6-311++G(d, p)$, with a MAE of $0.25 \mathrm{kcal} / \mathrm{mol}$, represents an overall better choice, while APFD/6-311++G(d,p), with a MAE of $0.86 \mathrm{kcal} / \mathrm{mol}$, greatly overestimated the interaction energy 


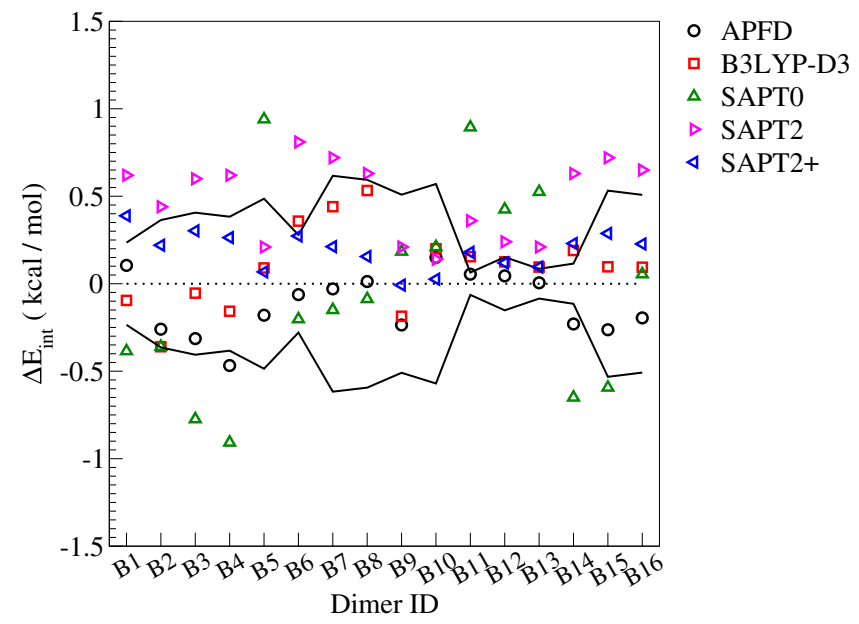

Fig. 2. Difference between the reference $\operatorname{CCSD}(\mathrm{T}) / \mathrm{CBS}$ energy and the results of the various methods tested, for the isocyanate test set (see Supplementary Material 1). Solid lines denote $10 \%$ accuracy w.r.t. the reference energies.

of both dispersion-dominated and strong H-bound complexes (see Supplementary Material 1 ).

For perturbative methods, a comparison on the isocyanate test set has been performed between the SAPT0/jun-cc-pvdz, SAPT2/augcc-pVDZ and SAPT2+/aug-cc-pVDZ, the last of which was suggested as a possible reference method for medium-size problems [49]. Higher order SAPT methods were precluded due to the size of the studied systems. It was found that SAPT0/jun-cc-pVDZ and SAPT2/aug-cc-pVDZ provide a similar, poor, accuracy. In particular, SAPT2 method systematically underestimates the interaction energies. In turn, SAPT2+ method provided much better results, with a MAE of $0.20 \mathrm{kcal} / \mathrm{mol}$ on the isocyanate set and of $0.34 \mathrm{kcal} / \mathrm{mol}$ for the S22 benchmark [49], making it the best available choice for SAPT calculations in this context.

\subsubsection{Interaction energies of model bimolecular complexes}

Isocyanurates interactions could be decomposed as the sum of independent contributions, that is $\mathrm{NCO}-\mathrm{NCO}(\mathrm{NN})$, NCO-isocyanurate

Table 1

Interaction energies (in $\mathrm{kcal} / \mathrm{mol}$ ) of model dimers, calculated at the B3LYP-D3BJ level of theory and using SAPT2/SAPT2+ methods.

\begin{tabular}{llllll}
\hline Dimer & ID & Contact type & $E_{D F T}$ & $E_{\text {SAPT2 }}$ & $E_{\text {SAPT2 }+}$ \\
\hline MIC-MIC & 1 & NCO-NCO & -5.81 & -5.36 & -5.89 \\
EIC-EIC & 2 & & -6.06 & -5.97 & -6.47 \\
EDI-EDI & 3 & & -6.68 & -6.60 & -7.17 \\
PIC-PIC & 4 & & -6.26 & -6.27 & -6.78 \\
PIC-GDI & 5 & & -7.18 & -7.08 & -7.61 \\
GDI-GDI & 6 & & -7.85 & -7.66 & -8.21 \\
HIC-HIC & 7 & & -6.37 & -6.44 & -6.98 \\
HIC-HDI & 8 & & -6.68 & -5.41 & -5.84 \\
HDI-HDI & 9 & & -6.92 & -6.86 & -7.45 \\
3MIC-MIC & 10 & NCO-ring & -7.08 & -7.07 & -7.55 \\
3MIC-EIC & 11 & & -7.38 & -7.62 & -8.11 \\
3MIC-PIC & 12 & & -7.71 & -7.96 & -8.46 \\
3EIC-EIC & 13 & & -7.02 & -7.33 & -7.91 \\
3GDI-3GDI & 14 & & -13.49 & $\mathrm{n} / \mathrm{a}$ & $\mathrm{n} / \mathrm{a}$ \\
3GDI-3GDI & 15 & & -17.58 & $\mathrm{n} / \mathrm{a}$ & $\mathrm{n} / \mathrm{a}$ \\
3MIC-3MIC & 16 & Ring-ring & -13.75 & -13.28 & -14.18 \\
A3MIC-A3MIC (a) & 17 & & -11.64 & -12.54 & -13.13 \\
A3MIC-A3MIC (b) & 18 & & -12.36 & -13.06 & -13.60 \\
A3MIC-3MIC & 19 & & -13.16 & -13.65 & -14.40 \\
3EIC-3EIC (a) & 20 & & -12.13 & -12.74 & -13.72 \\
3EIC-3EIC (b) & 21 & & -13.08 & -14.51 & -15.31 \\
3EIC-3EIC (c) & 22 & & -10.02 & -10.06 & -10.84 \\
\hline
\end{tabular}

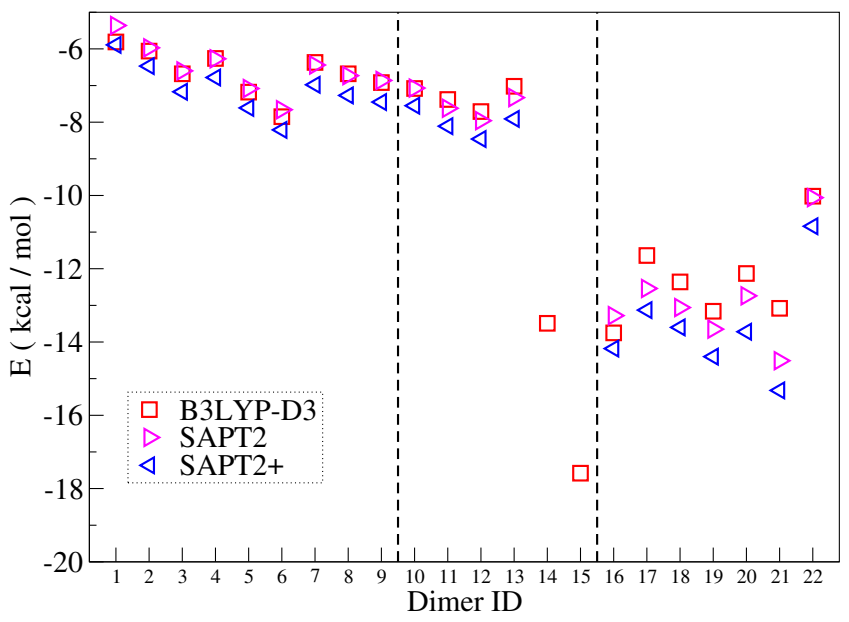

Fig. 3. Interaction energy for the dimers of Table 1 . The plot is subdivided by interaction type in NCO-NCO (NN) dimers, NCO-ring (NR) dimers and ring-ring (RR) dimers. Dimer IDs are the same as in Table 1.

ring (NR) and ring-ring (RR) contacts. Considering dimers of simple molecules, these interactions can be isolated, getting rid of the conformational complexity of larger systems, and their $E_{\text {int }}$ readily calculated. The molecules considered were methyl isocyanate (MIC), ethyl isocyanate (EIC) and ethylene diisocyanate (EDI), propyl isocyanate (PIC) and propylene diisocyanate (GDI), hexyl isocyanate (HIC) and hexamethylene diisocyanate (HDI), trimethyl symmetric (3MIC) and asymmetric (A3MIC) trimer, triethyl isocyanurate (3EIC) and propylene diisocyanate trimer (3GDI). The interaction energies of several dimers, whose optimized geometries can be found in Supplementary Material 3, calculated by DFT and SAPT methods are reported in Table 1 and Fig. 3.

The first group, which contains dimers involved in NN contacts, has the weakest $E_{\text {int }}$. The interaction energy seems to be weakly dependent on the alkylene chain length, while being influenced by the presence of NCO groups not directly involved in the contact, undergoing long-range electrostatic interactions. These effects are stronger for shorter molecules, in which the peripheral NCO groups are generally closer.

NR interactions, in the second group of Table 1, are a specific feature of systems containing both isocyanurate rings and isocyanate groups. For all the systems investigated, the geometrical optimization always led to a structure similar to that reported in Fig. 7: the $\mathrm{NCO}$ carbon adopts a bridge position with respect to the underlying ring $\mathrm{N}-\mathrm{C}$ bond, whereas the NCO oxygen places itself approximately along the ring axis. Their energy is of the same order of magnitude as NN contacts.

The strongest binding can be achieved with RR-type interactions, with $E_{\text {int }}$ always lower than $-10 \mathrm{kcal} / \mathrm{mol}$. The lowest interaction energies were obtained with a perfectly parallel stacking of the two molecules along the axis that passes through their ring centres, with a relative rotation of $\pi / 6$. This conformation is invariant under $\pi / 3$ rotations of one of the molecules around its axis. Longer alkylene chains extent always normally to the ring plane(i.e. either up or down), leading to several possible stable minima, all of which showed comparable energies.

A3MIC dimers provided slightly weaker $E_{\text {int }}$ than 3MIC dimers. The presence of two minima in Table 1 is due to the fact that the asymmetric ring dimer breaks the rotational symmetry and allows for two parallel stacked stable conformations, with different rotation angles.

Comparing DFT and SAPT results, it is evident that SAPT2/SAPT2+ energies are always lower than B3LYP ones, especially for RR-type 

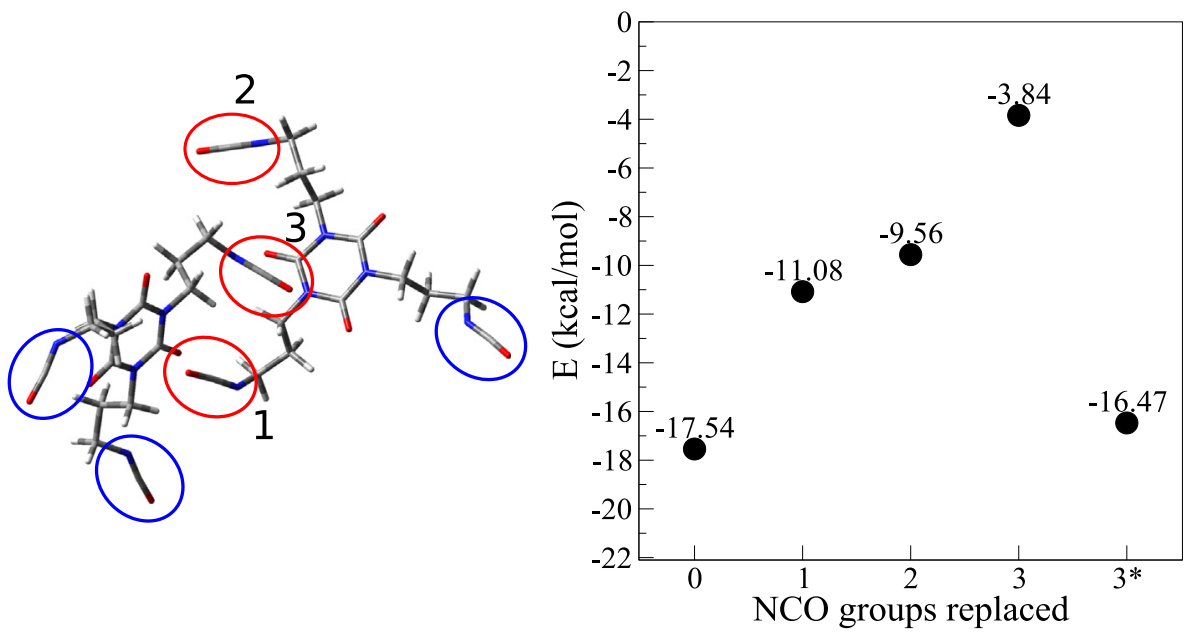

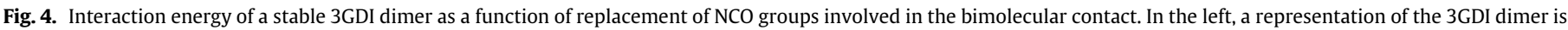

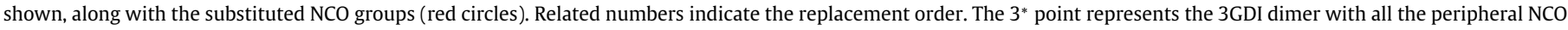
groups (blue circles) substituted. (For interpretation of the references to colour in this figure legend, the reader is referred to the web version of this article.)

interactions. Yet trends are in excellent agreement everywhere, except for the RR-type interaction branch, in which DFT indicates the 3MIC/3MIC dimer as the lowest energy one, in contrast with SAPT calculations. This discrepancy could be related to the difference in treatment of dispersion between these methods and higher level of theory calculations would be required to establish which predictions are more accurate.

Finally, to get an insight into the expected order of magnitude of the $E_{\text {int }}$ of larger molecules, the interaction energy of two stable conformations exhibiting NCO-ring interactions (see Fig. 4) of 3GDI isocyanurates was calculated. This revealed a relatively strong $E_{\text {int }}$, probably arising from the cooperation effect of different contributions, in which NR type interactions could play a crucial role. To verify this assumption, the most energetic 3GDI dimer was modified by sequentially replacing the NCO groups by a hydrogen atom, whose position was relaxed while keeping all other atoms fixed. In Fig. 4, along with the starting dimer representation, the resulting $E_{\text {int }}$ are reported.

The substitution of NCO groups labelled 1 and 3 increases the initial $E_{\text {int }}$ by 6.86 and $5.72 \mathrm{kcal} / \mathrm{mol}$, respectively. In contrast, the removal of the NCO group labelled 2 accounts for an increase in $E_{\text {int }}$ of $1.52 \mathrm{kcal} / \mathrm{mol}$ only. NCO groups 1 and 3 could then be identified as involved in NR contacts, accounting for $71.5 \%$ of $E_{\text {int }}$. This also proves the consistency of the interaction decomposition scheme proposed, as the residual contribution of the aliphatic chains and methane groups is small. Interestingly, if the distant NCO groups are substituted, only a small increase of $E_{\text {int }}$ of $1 \mathrm{kcal} / \mathrm{mol}$ can be observed, indicating that these groups bring a marginal contribution, likely only due to long-range electrostatic forces.

The data presented so far reveals that the isocyanurate ring, in both of its symmetric and asymmetric variants, is a strong noncovalent interaction site. In particular, it has been shown how it is capable of forming a perfectly stacked structure with a strong interaction energy, and to bind in a peculiar way with NCO groups. In fact, the NR contact seems to be the key mechanism to cooperatively build strong interaction energies between functional isocyanurate molecules.

\subsubsection{SAPT energy decomposition and $\mathrm{NCI}$ analysis}

The use of SAPT methods naturally provided a decomposition of the dimers interaction energy in terms of a clear physical meaning, allowing for a precise analysis of the nature of the different contacts. The decomposition of the attractive part of $E_{\text {int }}^{S A P T}$ for the studied dimers is reported in Fig. 5.

The SAPT energy decomposition revealed a net differentiation between NN contacts and the other types of contacts, with dispersion and electrostatic energies contributing almost equally to $E_{\text {int }}^{\text {SAPT }}$. Conversely, the dispersion energy accounts for $60-70 \%$ of the attractive part of $E_{\text {int }}^{S A P T}$ in NR contacts and, by a greater extent, in RR contacts. These calculations indicate that the interactions involving an isocyanurate ring are essentially similar, with the same prevalence of dispersion forces. The induction term provides a small contribution, being more relevant for NN and NR dimers, for which it accounts for $10-12 \%$ of the total attractive contribution.

Furthermore, an NCI analysis was performed on three model dimers, each representative of a contact type, to investigate the

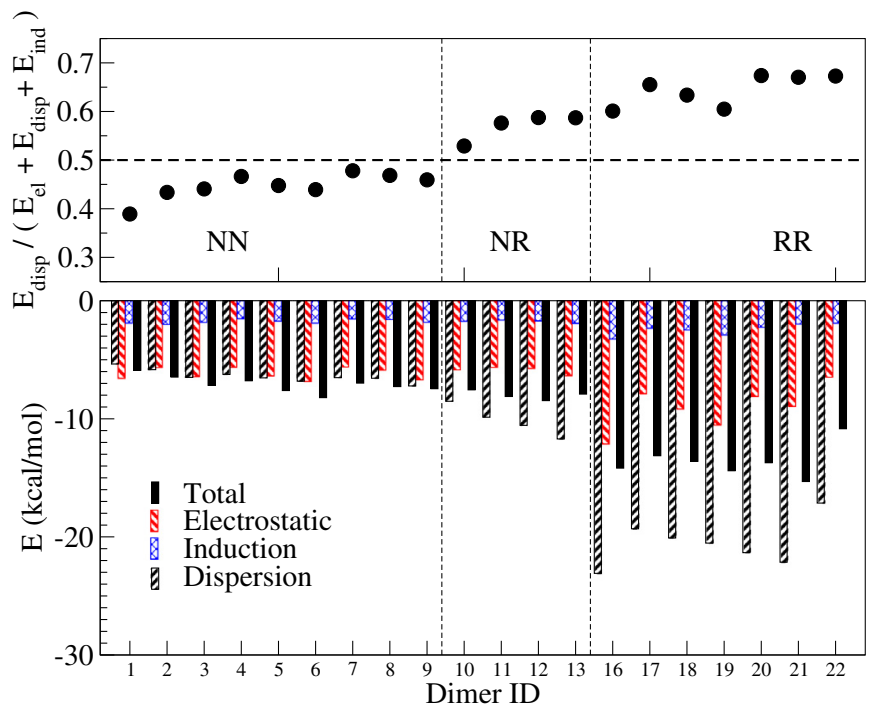

Fig. 5. bottom: Total $E_{\text {int }}^{S A P T}$ (black bar) and its attractive contributions, for the dimers of Table 1, calculated using the SAPT2+/aug-cc-pVDZ method. 3GDI trimers, too large for SAPT2 + calculations, are not reported. The plot follows the same subdivision in NCO-NCO (NN), NCO-ring (NR) and ring-ring dimers (RR) as Fig. 3. Top: ratio between the dispersion contribution and the attractive part of $E_{\text {int }}^{\text {SAPT }}$ for each dimer. 


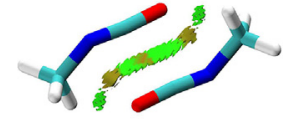

$\operatorname{sgn}\left(\lambda_{2}\right) \rho($ a. u.)

$$
\left[\begin{array}{r}
0.02 \\
0.01 \\
-0.00 \\
-0.01 \\
-0.02 \\
-0.03 \\
-0.04
\end{array}\right.
$$

Fig. 6. Reduced density gradient $s$ isosurfaces $(s=0.4)$ of methyl isocyanate dimer (top left), trimethyl isocyanurate stacked dimer (top right) and ethyl isocyanatetriethyl isocyanate dimer (bottom). The isosurfaces are coloured according to $\operatorname{sgn}\left(\lambda_{2}\right) \rho$ [38]. Intramolecular isosurfaces were omitted for clarity. (For interpretation of the references to colour in this figure legend, the reader is referred to the web version of this article.)

extension of the contact area and to gain further information on the nature of their interactions.

As it can be seen in Fig. 6, delocalized interactions are present, as expected for weak non-covalent interactions [38]. This is true in particular for NR and RR contacts, in which the interaction region is widespread and covers almost the entire isocyanurate ring. This indicates that the interactions involve a large area of the electron density above it, due to the $\pi$ orbitals normal to the ring plane from sp2 hybridized atoms. The $\mathrm{NCI}$ analysis suggests that ring-based dispersion forces are mostly caused by $\pi \cdots \pi$ interactions involving the $\pi$ orbitals of an isocyanurate ring interacting with, for example, those of a neighbouring isocyanate group or a second isocyanurate ring. The strong dispersion forces observed for the isocyanurate rings also suggest a possible use as an alternative for non-covalent functionalization of twodimensional materials.

\subsection{Local structure properties of isocyanate/isocyanurate liquids}

\subsubsection{Free energy calculations}

In previous sections, it was demonstrated that the different contact types between isocyanurates possess distinctive aspects. In particular, NR contacts showed very interesting features, such as a collaborative character. The objective of the next sections is to investigate the liquid state of isocyanate/isocyanurate systems to understand not only the way these molecules locally organize, but

\section{Table 2}

Free energies of binding of 3MIC and MIC dimers in the gas and liquid phase. Minimum distances refer to the gas phase. The zero value was set as the value of $G_{b}$ at $14 \AA$. The depth of the liquid MIC-3MIC minimum was measured on the metastable minimum at $3.9 \AA$.

\begin{tabular}{llll}
\hline Molecules & $r_{\min }(\AA)$ & $\Delta G_{b}^{\text {gas }}(\mathrm{kcal} / \mathrm{mol})$ & $\Delta G_{b}^{\text {liq }}(\mathrm{kcal} / \mathrm{mol})$ \\
\hline MIC-MIC & 3.9 & -0.94 & -0.18 \\
MIC-3MIC & 3.9 & -2.22 & $-0.10 *$ \\
3MIC-3MIC & 3.5 & -6.02 & -0.81 \\
\hline
\end{tabular}
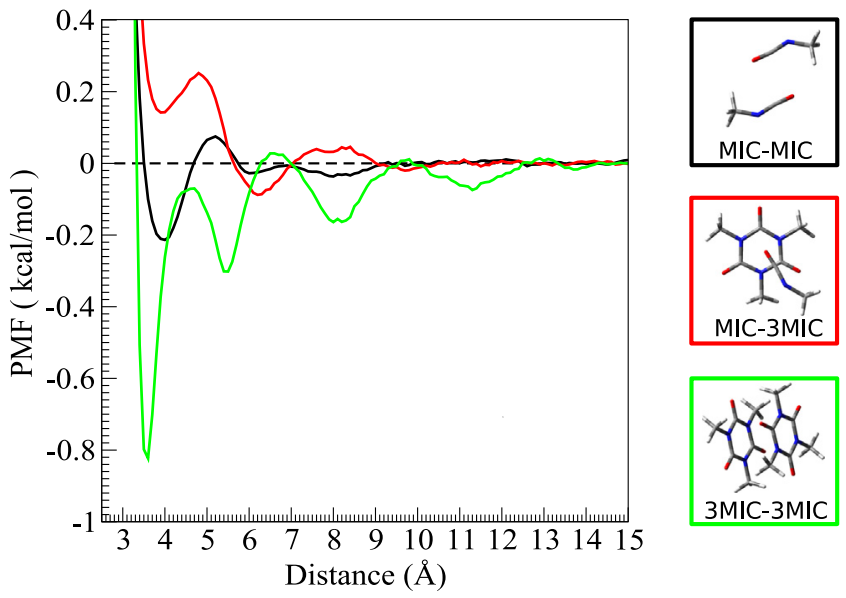

Fig. 7. PMF curves obtained at $298.15 \mathrm{~K}$ and $1 \mathrm{~atm}$, in a pure MIC liquid solution, for different possible contact types, exemplified by MIC-MIC (black), MIC-3MIC (red) and 3MIC-3MIC (green) dimers. The Jacobian term $k_{b} T \ln 2$ [42] was subtracted from all curves before plotting. (For interpretation of the references to colour in this figure legend, the reader is referred to the web version of this article.)

also the presence and stability of the discussed interactions and how they influence the local structure.

The free energy of binding $\Delta G_{b}$ provides information on the stability of a given dimer at non-zero temperature, and it was thus calculated, in gas and liquid phase, for the different contacts. The ring and NCO group geometric centres were taken as reference points, and their distance was defined as the collective variable for the $\mathrm{ABF}$ calculations. The liquid phase was simulated using a pure MIC liquid. This simplification, both in solution liquid and in the dimers involved, is necessary in order to obtain converged PMF curves in reasonable times, since more complex molecules would have required much longer simulations, because of their conformational complexity. Table 2 reports the $\Delta G_{b}^{\text {gas,liq }}$ for $3 \mathrm{MIC} / \mathrm{MIC}$ possible dimers, along with the minimum approach distance.

In the gas phase, there is only one free energy minimum that corresponds to the most stable structure. Interestingly, the MIC-3MIC dimer has a $\Delta G_{b}^{\text {gas }}$ that is twice than that of the MIC-MIC dimer, while the DFT-calculated interaction energies were equal. The strength of ring-ring interactions is confirmed here by the strong $\Delta G_{b}^{\text {gas }}$ of 3MIC$3 \mathrm{MIC}$ dimer. Thus, the different interactions can be clearly ordered by $\Delta G_{b}^{\text {gas }}$.

Concerning liquid phase calculations, the obtained PMF curves are reported in Fig. 7, showing a slightly different behaviour. The 3MIC-3MIC PMF curve exhibits several stable minima, with the closest (CM) being also the strongest, with a $\Delta G_{b}^{\text {liq }}$ of $-0.81 \mathrm{kcal} / \mathrm{mol}$, and corresponding to the parallel stacking conformation. In addition, there are two solvent separated minima at 5.4 and $8.2 \AA$, with a $\Delta G_{b}^{\text {liq }}$ of -0.22 and $-0.18 \mathrm{kcal} / \mathrm{mol}$, respectively. In the case of the MICMIC dimer, a relatively shallow PMF curve was found, with one weak minimum at $3.8 \AA$. The MIC-3MIC PMF curve, conversely, is more structured, showing one metastable minimum at $3.9 \AA$ and another around $6 \AA$.

$\mathrm{RR}$ contacts are dominant in liquid systems, due to their stronger $\Delta G_{b}^{\text {liq }}$, and the longer range, producing appreciable effects up to $12 \AA$. Concerning the other contacts types, the NN curve has a clear minimum, however the NR curve is not trivial, confirming that both could play a significant role in the liquid phase.

\subsubsection{Local structure characterization}

MD simulations of isocyanurate liquids were performed, considering different alkylene chain lengths containing $\mathrm{N}=3-6 \mathrm{CH}_{2}$ 


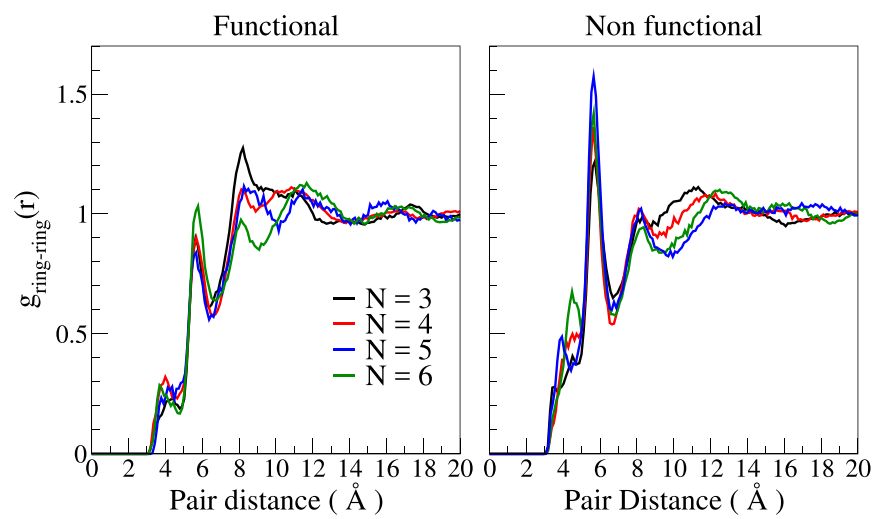

Fig. 8. Intermolecular ring-ring $g(r)$ for functional and non-functional isocyanate trimers, as a function of the ring-ring distance $r$. $N$ denotes the number of $-\left(\mathrm{CH}_{2}\right)-$ in the alkylene chains.

groups (see Fig. 1). Non-functional aliphatic isocyanurates were also included to study systems capable of RR contacts only. For each molecule, the ring geometric centre is the most convenient choice as reference point, and it was used to calculate the intermolecular ringring pair correlation function $g(r)$ on systems equilibrated at $298.15 \mathrm{~K}$ and $1 \mathrm{~atm}$ (Fig. 8). Furthermore, the $g(r)$ was decomposed by calculating, for each molecular pair, their relative tilt angle, that is the scalar product of their ring normal unit vectors, $\hat{n}$, according to

$\theta_{i j}=\cos ^{-1}\left(\hat{n}_{i} \cdot \hat{n}_{j}\right)$

in order to get the relative tilt angle distribution $g(r, \theta)$ (Fig. 9).

The ring-ring $g(r)$ shows several important features. Closest neighbours are located approximately at $3.5 \AA$, followed by a major peak around $6 \AA$, more pronounced for non-functional molecules. A third, smaller, peak can be identified at $8 \AA$, the height of which strongly depends on the alkylene chain length for functional trimers, while it is almost unchanged for non-functional ones. In the region above $10 \AA$, a weak modulation of the $g(r)$ around unity can be observed for both families. Regarding the $g(r, \theta)$, closest neighbours appear to stack, with a net majority of small angles. For the second peak, relative tilt angles are higher, indicating an abundance of tilted rings, that is in a " $T$ " conformation. At larger distances, it is impossible to extract useful information, as angular isotropy is recovered.

The analysis of $g(r)$ and $g(r, \theta)$ showed that RR interactions define the liquid microscopic structure, as already indicated by PMF calculations. Indeed, the $g(r)$ peaks and the PMF minima for the RR contacts correspond and their position is independent of either alkylene chain length or presence of NCO groups. Additionally, $g(r, \theta)$ showed that close rings prefers a parallel arrangement. Thus, it is expected that, in this region, RR contacts constitute the main intermolecular contact type. It is important to note that non-functional molecules display larger $g(r)$ at short distances, when compared to their functional counterparts. This indicates that, for the latter, RRtype interactions are partly screened. Based on the results presented in previous sections, this can be explained by the presence of NR contacts.

\subsubsection{Characterization of NR contacts}

As already mentioned, non-functional isocyanurates, at parity of conditions (alkylene chain length, temperature, pressure), typically exhibit viscosities that are 3-5 times lower compared to functional ones. In previous work, this was attributed [24] to the presence of NR-type interactions. Based on the low viscosity of linear diisocyanates, NN contacts are not expected to play a significant role. Therefore, the characterization of NR-type interactions in the liquid isocyanurates will help to verify the consistency of this hypothesis. In this regard, the distance $r_{c}$ between rings and -NCO group centres, belonging to different molecules, was measured for each possible pair and an NR contact was acknowledged if $r_{c}<4.0 \AA$. In Fig. 10, the radial NR contact density $n_{c}(r)$ is reported as a function of the intermolecular ring distance, normalized in such a way such that

$N_{N R}=\int_{0}^{\infty} 4 \pi r^{2} \mathrm{~d} r n_{c}(r)$

is the number of contact points per molecule.
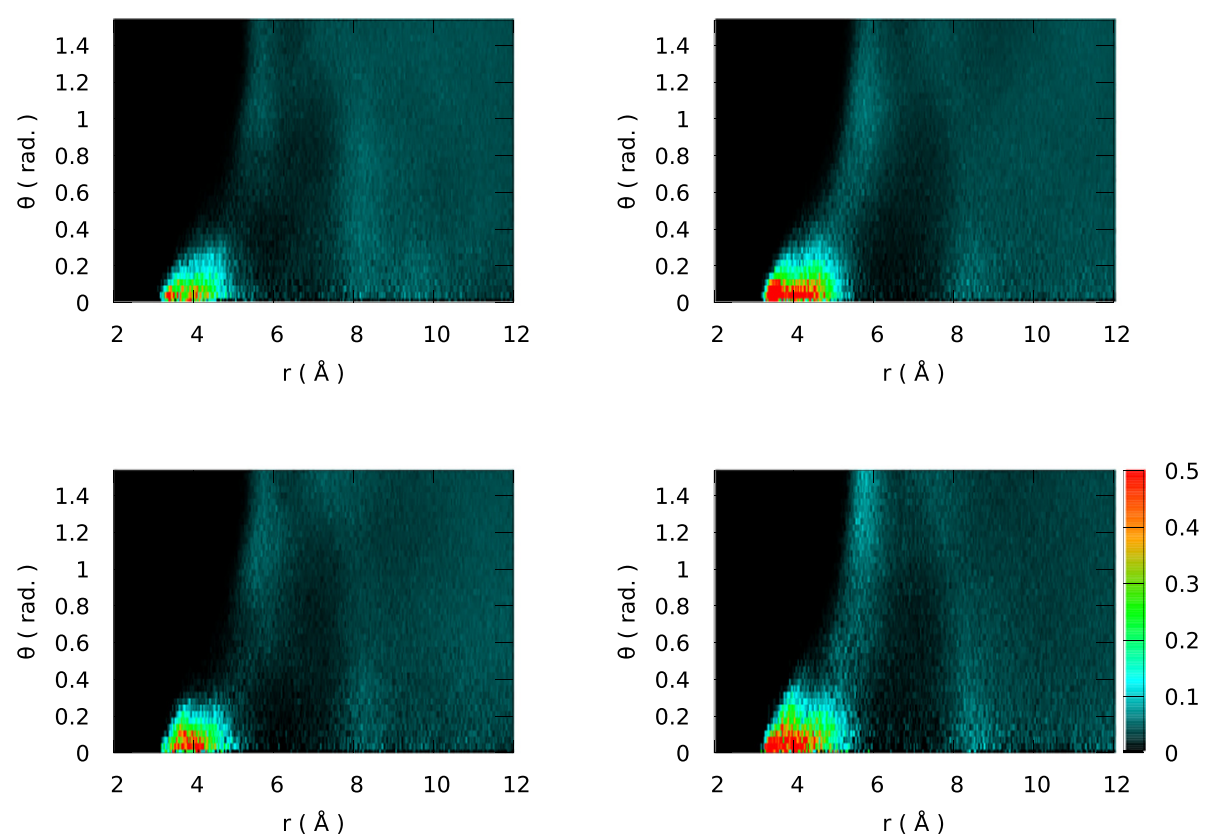

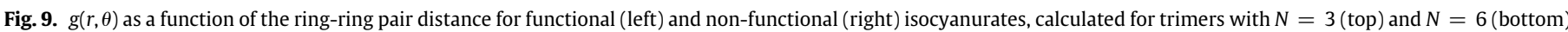
carbons in the alkylene chains. 


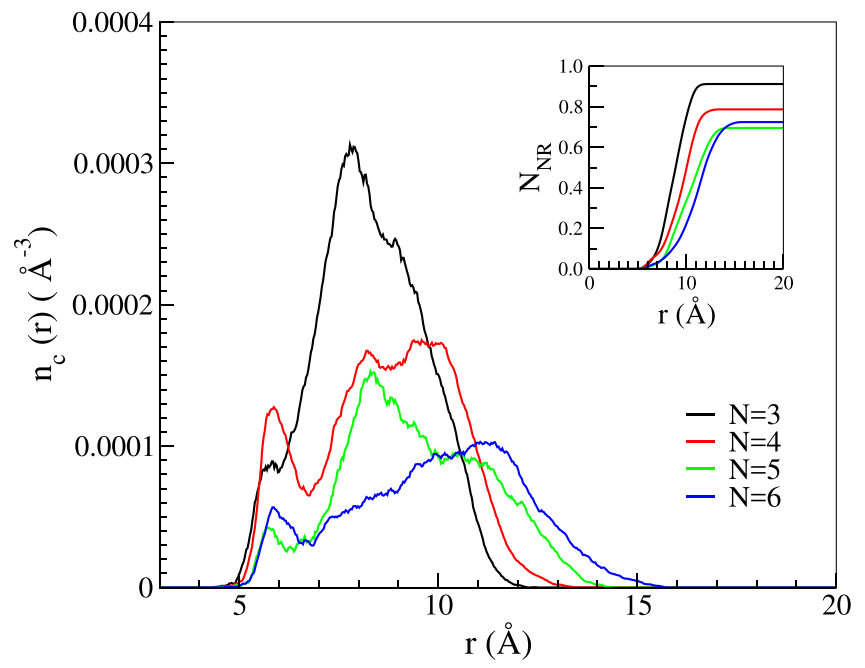

Fig. 10. Distribution $n_{c}(r)$ of NR contacts per molecule and per unit volume of functional isocyanurates as a function of the intermolecular ring-ring distance $r$, at $298.15 \mathrm{~K}$ and $1 \mathrm{~atm}$. $N$ denotes the number of $-\left(\mathrm{CH}_{2}\right)$ - in the alkylene chains.Inset: integral of $n_{c}(r)$, yielding the number $N_{N R}$ of NR contacts per molecule.

It can be observed that the NR contact density is greatly influenced by the alkylene chain length, in such a way that the maximum of the distribution decreases and the width increases, for increasing alkylene chain length. The only constant feature is the small peak at $6 \AA$, corresponding to the first $g(r)$ peak. The other peak at $8.2 \AA$, corresponding to the second $g(r)$ peak, is clearly visible for the lighter molecules, up to $N=5$, but then disappears for $N=6$, which displays a different contact distribution, more skewed towards larger distances. The difference could be due to the fact that, for short alkylene chains, terminal isocyanates are constrained in their movements while, in the opposite case, the space they can explore is much larger, hence a broader distribution of chain lengths is expected. At the same time, the probability of NR contacts at shorter distances is reduced, due to excluded volume effects.

The smallest system has $N_{N R}=0.9 \mathrm{NR}$ contacts per molecule, meaning that ca. $90 \%$ of the molecules are bound in this way. $N_{N R}$ seems to be proportional to the alkylene chain length, although there is a net change from $N=5$ to $N=6$, caused by the different character of their $n_{c}(r)$. It can be observed that $N_{N R}$ correlates with the experimental viscosity trends of functional isocyanurates [24], with higher values corresponding to higher viscosities.

In the range of short ring-ring distances, the NR contacts are absent and the interactions are dominated by the RR contacts, as already indicated by $g(r)$ and $g(r, \theta)$. However, at longer ranges, the RR contribution drops significantly, as dispersion forces decay more rapidly than dipole-dipole or any electrostatic interaction, and NR contacts provide the strongest intermolecular interaction energies. To prove this, a liquid phase simulation snapshot from the 3GDI liquid was analysed and, by using the ring-ring distance as radial variable, the DFT interaction energy of all pairs at a fixed distance, within a bin $0.1 \AA$ wide, was calculated. Because of the large number of calculations on large systems ( 90 atoms), the basis set was reduced to $6-31+G(d, p)$. The results, divided according to the presence or absence of NR contacts, are collected in Table 3. Although they occur less frequently, in each bin, NR-bound pairs on average have a stronger $E_{\text {int }}$ than non-NR bound pairs, roughly by a factor 2. This proves that the presence of NR contacts helps to strengthen the cohesive forces in the liquid, because they add a significant contribution to the intermolecular interaction energies.
Table 3

Averaged DFT interaction energies of all intermolecular pairs at the indicated ring-ring distance, for a 3GDI liquid at $298.15 \mathrm{~K}$ and $1 \mathrm{~atm}$. In each bin, the pairs were divided in two complementary sets, according to the presence or absence of NR contacts.

\begin{tabular}{lllll}
\hline Distance $(\AA)$ & NR pairs & $E_{\text {int }}(\mathrm{kcal} / \mathrm{mol})$ & No NR pairs & $E_{\text {int }}(\mathrm{kcal} / \mathrm{mol})$ \\
\hline $6.65 \pm 0.05$ & 5 & $-10.7 \pm 4.0$ & 10 & $-6.0 \pm 1.6$ \\
$7.65 \pm 0.05$ & 13 & $-11.0 \pm 2.5$ & 34 & $-5.9 \pm 2.2$ \\
$8.65 \pm 0.05$ & 14 & $-8.8 \pm 2.6$ & 49 & $-4.7 \pm 1.8$ \\
\hline
\end{tabular}

\section{Conclusions}

In this work, intermolecular interactions between isocyanurates and isocyanates have been studied in detail, both using $a b$ initio methods and liquid-phase MD simulations. The nature of intermolecular interactions of aliphatic isocyanurate has been clarified, as well as their effect in the microscopic structure of isocyanurate liquids.

It was shown that isocyanate groups only weakly interact with one another through a combination of electrostatic interactions caused by their dipole moments and, as indicated by SAPT calculations, dispersion forces. Isocyanurate rings on the other hand were proven to be strong non-covalent interaction sites, being capable of inducing large intermolecular interaction energies as stacked dimers. This interaction is dominated by dispersion contributions, arising from the many $\pi$ orbitals perpendicular to the ring plane. Such strong dispersion character suggests a possible application of isocyanurates as non-covalent functionalization agents of two-dimensional materials. Moreover, ring-ring interactions modulate the microscopic structure of isocyanurate liquids, either functional or not.

The third interaction type that was investigated, namely between isocyanates and isocyanurates, lies in between the other two, and turned out to be the key to understanding the viscosity of functional isocyanurate liquids. This interaction is of the same order of magnitude as the isocyanate-isocyanate interactions, but, unlike them, is dispersion-dominated because of the $\pi \cdots \pi$ interactions with the isocyanurate ring. In functional systems, containing both isocyanates and isocyanurates, this leads to two effects. At short distances, the presence of isocyanate-isocyanurate contacts reduces the occurrences of ring-ring stacked assemblies. At longer distances, the presence of isocyanate-isocyanurate contacts increases the intermolecular interaction energies by means of cooperative effects, in conjunction to the isocyanate dipole-dipole interactions. The second effect, long distance in nature, causes a significant increase in the cohesive forces in the liquid state, leading to increased viscosity. Thereby, it was shown that it is possible to relate the viscosity of isocyanate-isocyanurate liquids to the density of isocyanateisocyanurate (NR) contacts.

\section{Acknowledgments}

This project has received funding from the European Union's Horizon 2020 - Research and Innovation Framework Programme under the Marie Skłodowska-Curie Grant Agreement no. 642890 (http://thelink-project.eu/) and it was partially supported by the Portuguese Science and Technology Foundation (FCT) in the framework of the Strategic Funding UID/FIS/04650/2013, by the FCT grant SFRH/BD/128666/2017 and by the project " SearchON2: Revitalization of HPC infrastructure of Uminho" (NORTE07-0162-FEDER-0000869), under the National Strategic Reference Framework, through the European Regional Development Fund. The authors also acknowledge the Texas Advanced Computing Center (TACC) at The University of Texas at Austin for providing HPC resources that have contributed to the research results reported within this paper. 


\section{Appendix A. Supplementary data}

Supplementary data to this article can be found online at https:// doi.org/10.1016/j.molliq.2019.01.165.

\section{References}

[1] F.E. Golling, R. Pires, A. Hecking, J. Weikard, F. Richter, K. Danielmeier, D. Dijkstra, Polyurethanes for coatings and adhesives - chemistry and applications, Polym. Int. (2018) https://doi.org/10.1002/pi.5665.

[2] H.-J. Kang, E.J. Cha, H.-D. Park, Protein immobilization onto various surfaces using a polymer-bound isocyanate, Appl. Surf. Sci. 324 (Supplement C) (2015) 198-204. http://www.sciencedirect.com/science/article/pii/ S0169433214023605. https://doi.org/10.1016/j.apsusc.2014.10.117.

[3] J.A. Howarter, J.P. Youngblood, Self-cleaning and anti-fog surfaces via stimuliresponsive polymer brushes, Adv. Mater. 19 (22) (2007) 3838-3843. https:// doi.org/10.1002/adma.200700156.

[4] A.R. McDonald, H.P. Dijkstra, B.M.J.M. Suijkerbuijk, G.P.M. van Klink, G. van Koten, "Click" immobilization of organometallic pincer catalysts for C-C coupling reactions, Organometallics 28 (16) (2009) 4689-4699. https://doi.org/10. 1021/om900237g.

[5] S. Kuypers, S.K. Pramanik, L. D’Olieslaeger, G. Reekmans, M. Peters, J. D’Haen, D. Vanderzande, T. Junkers, P. Adriaensens, A. Ethirajan, Interfacial thiolisocyanate reactions for functional nanocarriers: a facile route towards tunable morphologies and hydrophilic payload encapsulation, Chem. Commun. 51 (2015) 15858-15861. https://doi.org/10.1039/C5CC05258K.

[6] F. Borbone, A. Carella, A. Roviello, M. Casalboni, F. De Matteis, G. Stracci, F. Della Rovere, A. Evangelisti, M. Dispenza, Outstanding poling stability of a new cross-linked nonlinear optical (NLO) material from a low molecular weight chromophore, J. Phys. Chem. B 115 (42) (2011) 11993-12000. https://doi.org/ 10.1021/jp204545k.

[7] R.M. Arnold, D.L. Patton, V.V. Popik, J. Locklin, A dynamic duo: pairing click chemistry and postpolymerization modification to design complex surfaces, Acc. Chem. Res. 47 (10) (2014) 2999-3008. https://doi.org/10.1021/ ar500191m.

[8] M. Huo, N. Wang, T. Fang, M. Sun, Y. Wei, J. Yuan, Single-chain polymer nanoparticles: mimic the proteins, Polymer (United Kingdom) 66 (2015) A11A21. https://doi.org/10.1016/j.polymer.2015.04.011.

[9] G. Gody, C. Rossner, J. Moraes, P. Vana, T. Maschmeyer, S. Perrier, Onepot RAFT/“Click" chemistry via isocyanates: efficient synthesis of alpha-endfunctionalized polymers, J. Am. Chem. Soc. 134 (30) (2012) 12596-12603. https://doi.org/10.1021/ja3030643.

[10] G. Gody, D.A. Roberts, T. Maschmeyer, S. Perrier, A new methodology for assessing macromolecular click reactions and its application to amine-tertiary isocyanate coupling for polymer ligation, J. Am. Chem. Soc. 138 (12) (2016) 4061-4068. https://doi.org/10.1021/jacs.5b11831.

[11] P. Sundberg, M. Karppinen, Organic and inorganic-organic thin film structures by molecular layer deposition: a review, Beilstein J. Nanotechnol. 5 (2014) 1104-1136. https://doi.org/10.3762/bjnano.5.123.

[12] S. Stankovich, R.D. Piner, S.B.T. Nguyen, R.S. Ruoff, Synthesis and exfoliation of isocyanate-treated graphene oxide nanoplatelets, Carbon 44 (15) (2006) 33423347. https://doi.org/10.1016/j.carbon.2006.06.004.

[13] A. Kafy, K.K. Sadasivuni, H.-C. Kim, A. Akther, J. Kim, Designing flexible energy and memory storage materials using cellulose modified graphene oxide nanocomposites, Phys. Chem. Chem. Phys. 17 (8) (2015) 5923-5931. https:// doi.org/10.1039/C4CP05921B.

[14] M. Dekamin, F. Mehdipoor, A. Yaghoubi, 1,3,5-Tris(2hydroxyethyl)isocyanurate functionalized graphene oxide: a novel and efficient nanocatalyst for the one-pot synthesis of 3,4-dihydropyrimidin-2(1: H)-ones, New J. Chem. 41 (14) (2017) 6893-6901. https://doi.org/10.1039/ c7nj00632b.

[15] M. Müller, Q.U. Huynh, E. Uhlmann, M.H. Wagner, Study of inkjet printing as additive manufacturing process for gradient polyurethane material, Prod. Eng. 8 (1-2) (2014) 25-32. https://doi.org/10.1007/s11740-013-0504-0.

[16] S.L. Cooper, J. Guan (Eds.), Advances in Polyurethane Biomaterials, Woodhead Publishing, 2016

[17] T.A.C. Flipsen, R. Steendam, A.J. Pennings, G. Hadziioannou, A novel thermoset polymer optical fiber, Adv. Mater. 8 (1) (1996) 45-48. https://doi.org/10.1002/ adma.19960080107.

[18] P. Fabbri, S.M. Poor, L. Ferrari, L. Rovati, S. Borsacchi, M. Geppi, P. Lima, L. Carlos, Highly stable plastic optical fibre amplifiers containing $[\mathrm{Eu}(\mathrm{btfa}) 3(\mathrm{MeOH})($ bpeta)]: a luminophore able to drive the synthesis of polyisocyanates, Polymer 55 (2) (2014) 488-494. http://www. sciencedirect.com/science/article/pii/S0032386113011439. https://doi.org/10. 1016/j.polymer.2013.12.043.

[19] D. Chattopadhyay, K. Raju, Structural engineering of polyurethane coatings for high performance applications, Prog. Polym. Sci. 32 (3) (2007) 352-418. http:// www.sciencedirect.com/science/article/pii/S0079670006001365. https://doi. org/10.1016/j.progpolymsci.2006.05.003.

[20] M. Moritsugu, A. Sudo, T. Endo, Cyclotrimerization of diisocyanates toward high-performance networked polymers with rigid isocyanurate structure: combination of aromatic and aliphatic diisocyanates for tunable flexibility, J. Polym. Sci. A Polym. Chem. 51 (12) (2013) 2631-2637. https://doi.org/10.1002/ pola.26651.
[21] H. Renz, B. Bruchmann, Pathways targeting solvent-free \{PUR \} coatings, Prog. Org. Coat. 43 (1-3) (2001) 32-40. http://www.sciencedirect. com/science/article/pii/S0300944001002399. https://doi.org/10.1016/S03009440(01)00239-9.

[22] E. Preis, N. Schindler, S. Adrian, U. Scherf, Microporous polymer networks made by cyclotrimerization of commercial, aromatic diisocyanates, ACS Macro Lett. 4 (11) (2015) 1268-1272. https://doi.org/10.1021/acsmacrolett. 5 b00726.

[23] S. Donthula, C. Mandal, T. Leventis, J. Schisler, A.M. Saeed, C. Sotiriou-Leventis, N. Leventis, Shape memory superelastic poly(isocyanurate-urethane) aerogels (PIR-PUR) for deployable panels and biomimetic applications, Chem. Mater. 29 (10) (2017) 4461-4477. https://doi.org/10.1021/acs.chemmater.7b01020.

[24] P.J. Driest, V. Lenzi, L.S.A. Marques, M.M.D. Ramos, D.J. Dijkstra, F.U. Richter, D. Statamatialis, D.W. Grijpma, Aliphatic isocyanurate and polyisocyanate networks, Polym. Adv. Technol. 28 (10) (2017) 1299-1304. https://doi.org/10. 1002/pat.3891.

[25] European Chemicals Agency, (2018) last time visited: 27th September 2018. https://echa.europa.eu.

[26] M.J. Frisch, G.W. Trucks, H.B. Schlegel, G.E. Scuseria, M.A. Robb, J.R. Cheeseman, G. Scalmani, V. Barone, B. Mennucci, G.A. Petersson, H. Nakatsuji, M. Caricato, X. Li, H.P. Hratchian, A.F. Izmaylov, J. Bloino, G. Zheng, J.L. Sonnenberg, M. Hada, M. Ehara, K. Toyota, R. Fukuda, J. Hasegawa, M. Ishida, T. Nakajima, Y. Honda, O. Kitao, H. Nakai, T. Vreven, J.A. Montgomery, Jr., J.E. Peralta, F. Ogliaro, M. Bearpark, J.J. Heyd, E. Brothers, K. N. Kudin, V. N. Staroverov, R. Kobayashi, J. Normand, K. Raghavachari, A. Rendell, J.C. Burant S.S. Iyengar, J. Tomasi, M. Cossi, N. Rega, J. M. Millam, M. Klene, J.E. Knox, J.B. Cross, V. Bakken, C. Adamo, J. Jaramillo, R. Gomperts, R.E. Stratmann, O. Yazyev, A.J. Austin, R. Cammi, C. Pomelli, J.W. Ochterski, R.L. Martin, K. Morokuma, V.G. Zakrzewski, G.A. Voth, P. Salvador, J.J. Dannenberg, S. Dapprich, A.D. Daniels, Ö. Farkas, J.B. Foresman, J.V. Ortiz, J. Cioslowski, D.J. Fox, Gaussian '09 Revision E.01, 2009, Gaussian Inc., Wallingford CT.

[27] A.D. Becke, Density-functional thermochemistry. III. The role of exact exchange, J. Chem. Phys. 98 (7) (1993) 5648-5652. http://scitation.aip.org/content/aip/ journal/jcp/98/7/10.1063/1.464913. https://doi.org/10.1063/1.464913.

[28] A. Austin, G.A. Petersson, M.J. Frisch, F.J. Dobek, G. Scalmani, K. Throssell, A density functional with spherical atom dispersion terms, J. Chem. Theory Comput 8 (12) (2012) 4989-5007. https://doi.org/10.1021/ct300778e.

[29] S. Grimme, J. Antony, S. Ehrlich, H. Krieg, A consistent and accurate ab initio parametrization of density functional dispersion correction (DFT-D) for the 94 elements H-Pu, J. Chem. Phys. 132 (15) (2010) 1-19. http://scitation.aip.org/ content/aip/journal/jcp/132/15/10.1063/1.3382344. https://doi.org/10.1063/1. 3382344.

[30] S. Grimme, S. Ehrlich, L. Goerigk, Effect of the damping function in dispersion corrected density functional theory, Journal of Computational Chemistry 32 (7) (2011) 1456-1465. https://doi.org/10.1002/jcc.21759.

[31] T.H. Dunning, Gaussian basis sets for use in correlated molecular calculations. I. The atoms boron through neon and hydrogen, J. Chem. Phys. 90 (2) (1989) 1007-1023. https://doi.org/10.1063/1.456153.

[32] R. Ditchfield, W.J. Hehre, J.A. Pople, Self-consistent molecular-orbital methods. IX. An extended Gaussian-type basis for molecular-orbital studies of organic molecules, J. Chem. Phys. 54 (2) (1971) 724-728. http://scitation.aip. org/content/aip/journal/jcp/54/2/10.1063/1.1674902. https://doi.org/10.1063/ 1.1674902.

[33] M.J. Frisch, J.A. Pople, J.S. Binkley, Self-consistent molecular orbital methods 25. Supplementary functions for Gaussian basis sets, J. Chem. Phys. 80 (7) (1984) 3265-3269. http://scitation.aip.org/content/aip/journal/jcp/80/7/10. 1063/1.447079. https://doi.org/10.1063/1.447079.

[34] T. Clark, J. Chandrasekhar, G.W. Spitznagel, P.V.R. Schleyer, Efficient diffuse function-augmented basis sets for anion calculations. III. The $3-21+G$ basis set for first-row elements, Li-F, J. Comput. Chem. 4 (3) (1983) 294-301. https://doi. org/10.1002/jcc.540040303.

[35] S. Boys, F. Bernardi, The calculation of small molecular interactions by the differences of separate total energies. Some procedures with reduced errors, Mol. Phys. 19 (4) (1970) 553-566. https://doi.org/10.1080/00268977000101561.

[36] K. Szalewicz, Symmetry-adapted perturbation theory of intermolecular forces, Wiley Interdiscip. Rev.: Comput. Mol. Sci. 2 (2) (2012) 254-272. https://doi.org/ $10.1002 /$ wcms.86.

[37] R.M. Parrish, L.A. Burns, D.G.A. Smith, A.C. Simmonett, A.E. DePrince, E.G. Hohenstein, U. Bozkaya, A.Y. Sokolov, R. Di Remigio, R.M. Richard, J.F. Gonthier, A.M. James, H.R. McAlexander, A. Kumar, M. Saitow, X. Wang, B.P. Pritchard, P. Verma, H.F. Schaefer, K. Patkowski, R.A. King, E.F. Valeev, F.A. Evangelista, J.M. Turney, T.D. Crawford, C.D. Sherrill, Psi4 1.1: An open-source electronic structure program emphasizing automation, advanced libraries, and interoperability, J. Chem. Theory Comput. 13 (7) (2017) 3185-3197. https://doi.org/10. 1021/acs.jctc.7b00174.

[38] C. Narth, Z. Maroun, R.A. Boto, R. Chaudret, M.-L. Bonnet, J.-P. Piquemal, J Contreras-García, A complete NCI perspective: from new bonds to reactivity, Applications of Topological Methods in Molecular Chemistry, 2016, pp. 491527. http://link.springer.com/10.1007/978-3-319-29022-5. https://doi.org/10. 1007/978-3-319-29022-5_18.

[39] Multiwfn, A Multifunctional Wavefunction Analyzer, last time visited: 27th July 2018. http://sobereva.com/multiwfn/.

[40] S. Plimpton, Fast parallel algorithms for short-range molecular dynamics, Journal of Computational Physics 117 (1) (1995) 1-19. http://www. sciencedirect.com/science/article/pii/S002199918571039X. https://doi.org/10. 1006/jcph.1995.1039. 
[41] V. Lenzi, P.J. Driest, D.J. Dijkstra, M.M.D. Ramos, L.S.A. Marques, GAFF-IC: realistic viscosities for isocyanate molecules with a GAFF-based force field Mol. Simul. 45 (3) (2019) 207-214. https://doi.org/10.1080/08927022.2018. 1554902.

[42] G. Fiorin, M. Klein, J. Hénin, Using collective variables to drive molecular dynamics simulations, Mol. Phys. (2013) https://doi.org/10.1080/00268976. 2013.813594

[43] W.G. Hoover, Canonical dynamics: equilibrium phase-space distributions, Phys. Rev. A 31 (1985) 1695-1697. https://doi.org/10.1103/PhysRevA.31.1695.

[44] M. Parrinello, A. Rahman, Polymorphic transitions in single crystals: a new molecular dynamics method, J. Appl. Phys. 52 (12) (1981) 7182-7190. http:/ scitation.aip.org/content/aip/journal/jap/52/12/10.1063/1.328693. https://doi org/10.1063/1.328693.

[45] S.J. Plimpton, R. Pollock, M. Stevens, Particle-mesh Ewald and rRESPA for Parallel Molecular Dynamics Simulations, PPSC, 1997.
[46] R.E. Isele-Holder, W. Mitchell, A.E. Ismail, Development and application of a particle-particle particle-mesh Ewald method for dispersion interactions, J. Chem. Phys. 137 (17) (2012) 174107. https://doi.org/10.1063/1.4764089.

[47] T. Takatani, E.G. Hohenstein, M. Malagoli, M.S. Marshall, C.D. Sherrill, Basis set consistent revision of the S22 test set of noncovalent interaction energies, J. Chem. Phys. 132 (14). (2010) https://doi.org/10.1063/1.3378024.

[48] P. Jurečka, J. Šponer, J. Černý, P. Hobza, Benchmark database of accurate (MP2 and $\operatorname{CCSD}(\mathrm{T})$ complete basis set limit) interaction energies of small model complexes, DNA base pairs, and amino acid pairs, Phys. Chem. Chem. Phys. 8 (17) (2006) 1985-1993. http://xlink.rsc.org/?DOI=B600027D. https://doi.org/ 10.1039/B600027D.

[49] T.M. Parker, L.A. Burns, R.M. Parrish, A.G. Ryno, C.D. Sherrill, Levels of symmetry adapted perturbation theory (SAPT). I. Efficiency and performance for interaction energies, J. Chem. Phys. 140 (9). (2014) https://doi.org/10.1063/1. 4867135. 